Y. Kitaoka

Nagoya Math. J.

Vol. 115 (1989), 23-41

\title{
SOME REMARKS ON REPRESENTATIONS OF POSITIVE DEFINITE QUADRATIC FORMS
}

\author{
YOSHIYUKI KITAOKA
}

Let $S, T$ be positive definite integral symmetric matrices of degree $m, n$ respectively and let us consider the quadratic diophantine equation $S[X]=T$. We know already [1] that the following assertion $(A)_{m, n}$ is true for $m \geq 2 n+3$.

$(A)_{m, n}$ : There exists a constant $c(S)$ such that $S[X]=T$ has an integral solution $X \in M_{m, n}(Z)$ if $S[X]=T$ has an integral solution $X €$ $M_{m, n}\left(Z_{p}\right)$ for every prime $p$ and $\min T>c(S)$.

In the above, min $T$ denotes the minimum of $T[x]$ for all non-zero integral vectors $x$. The basic question is whether the number $2 n+3$ is best possible or not. As facts which suggest that $2 n+3$ is best, we can enumerate the following (i), (ii), (iii):

(i) When $n=1$, it is the case.

(ii) From the quantitative viewpoint, the Siegel's weighted average of the numbers of solutions of $S_{i}[X]=T$ where $S_{i}$ runs over a complete set of representatives of the classes in the genus of $S$, is expected to be not few if $(A)_{m, n}$ is true. By a Siegel's formula [9], the weighted average is $|T|^{(m-n-1) / 2}$ times the infinite product of local densities $\alpha_{p}(s, T)$ up to the elementary constant depending only on $S$ and $n$, and it is known [2] that there is a positive constant $c_{1}(s)$ such that the infinite product of local densities is larger than $c_{1}(S)$ as far as $T$ is represented by $S$ over $Z_{p}$ for every prime $p$ if and only if $m \geq 2 n+3$.

(iii) The condition $m \geq 2 n+3$ appears often naturally at an analytic approach.

Next, let us look at the problem from another viewpoint which leads us to the suggestion incompatible with the above observation for $n>1$. It is known [2] that $(A)_{m, n}$ does not hold for $m=n+3$. It is the best for all $n$ till now, as far as the author knows. When $m=n+3$, we

Received June 9, 1988. 
constructed counterexamples by the following idea. Suppose $S[X]=T$ for $X \in M_{m, n}(Z)$; writing $X=Y Z$ with a primitive matrix $Y \in M_{m, n}(Z)$ and $Z \in M_{n, n}(Z), \bar{T}:=T\left[Z^{-1}\right]=S[Y]$ is (primitively represented globally by $S$ and hence) primitively represented by $S$ over $Z_{p}$, and it yields that $\min \bar{T}$ is less than $\min S$. This is a contradiction.

Now the following problem emerges along this line: Let $S, T m, n$ be those as above, $S[X]=T$ is soluble over $Z_{p}$ for every prime $p$, and $\min T$ is large. Then for every matrix $\bar{T}$ which satisfies

(i) $S[X]=\bar{T}$ has a primitive solution over $Z_{p}$ for every prime $p$, and

(ii) $\bar{T}[X]=T$ is soluble for $X \in M_{n, n}(Z)$, is $\min \bar{T}$ small?

We have obtained counterexamples for $m=n+3$ by showing the affirmative of this question. If it is affirmative for $m=2 n+2$, then, reforming $S$, we must construct a counterexample for $(A)_{2 n+2, n}$. When $m=2 n+2$ and $n=1$, it is affirmative and we have a counterexample for $(A)_{4,1}$. However it turns out to be negative for $m=2 n+2, n \geq 2$, which is an aim of this paper, that is the following assertion $(R)_{m, n}$ is true for $m=2 n+2, n \geq 2$ (Theorem in 1 in the text):

$(R)_{m, n}$ : Let $S, T m, n$ be those as above and suppose that $S[X]=T$ is soluble over $Z_{p}$ for every prime $p$. Then there exists a positive integral matrix $\bar{T}$ of degree $n$ satisfying

(i ) $S[X]=\bar{T}$ has a primitive solution $X$ over $Z_{p}$ for every prime $p$,

(ii) $\bar{T}[X]=T$ is soluble for $X \in M_{n, n}(Z)$, and

(iii) if $\min T$ is large, then $\min \bar{T}$ is also large.

Moreover in connection with primitiveness in (i), let us consider the following assertions:

$(A P)_{m, n}$ : There exists a constant $c^{\prime}(S)$ such that $S[X]=T$ has a primitive integral solution $X \in M_{m, n}(Z)$ if $S[X]=T$ has a primitive integral solution $X \in M_{m, n}\left(Z_{p}\right)$ for every prime $p$ and $\min T>c^{\prime}(S)$.

$(A P W)_{m, n}$ : The weaker assertion than $(A P)_{m, n}$ which does not require the primitiveness of global solution.

Since $(A)_{2 n+3, n}$ is true and $(A P W)_{m, n}$ has a stronger assumption than $(A)_{m, n}$, one may expect the validity of $(A P W)_{2 n+2, n}$ or strongly $(A P)_{2 n+2, n}$, taking account of the validity of $(A P)_{4,1}$ and hence $(A P W)_{4,1}$. The weak assertion $(A P W)_{2 n+2, n}$ implies the assertion $(A)_{2 n+2, n}$ by virtue of the validity of $(R)_{2 n+2, n}$ for $n \geq 2$. If, hence $(A)_{2 n+2, n}$ is false for $n \geq 2$, then 
$(A P)_{2 n+2, n}$ and $(A P W)_{2 n+2, n}$ are also false. Here we note again that $(R)_{4,1}$ is false and it yields immediately the falsehood of $(A)_{4,1}$ but $(A P)_{4,1}$ (and hence $\left.(A P W)_{4,1}\right)$ is true. Results here and [3], [5], [6] may suggest the validity of $(A)_{2 n+2, n}$ for $n \geq 2$. This dennies the suggestion at the beginning that $2 n+3$ is best possible for $n \geq 2$. Which is plausible? In 2 in the text, we show that $(R)_{m, n}(m \geq n+3$ and $n \geq 3)$ is valid for scalings of a fixed $T_{0}$ with small limitation. It shows that it is hard to construct counterexamples for $(A)_{m, n}$ for $m \geq n+3, n \geq 3$ by a special sequence of $T$ which are scalings of some fixed $T_{0}$.

Let us discuss the case of $m=2 n+2 \geq 6$ from the analytic viewpoint in passing. We put a fundamental assumption that for every Siegel modular form $f(Z)=\sum a(T) \exp (2 \pi i \operatorname{tr} T Z)$ of degree $n$, weight $n+1$ and some level, whose constant term vanishes at each cusp, the estimate $a(T)=O\left((\min T)^{-\varepsilon}|T|^{(n+1) / 2}\right)$ holds for some positive $\varepsilon$ if $\min T$ is larger than some constant independent of $f(Z)$. To verify the assertion $(A)_{2 n+2, n}$ it is sufficient to do the assertion $(A P W)_{2 n+2, n}$ as above. Suppose that $S[X]=T$ has a primitive solution $X=X_{p} \in M_{m, n}\left(Z_{p}\right)$ for every prime $p$. Let $r_{\mathrm{pr}}(T, S)$ be the number of integral primitive solutions of $S[X]=T$. As in $\S 1.7$ in [3] we have

$$
r_{\mathrm{pr}}(T, S)=S W_{p}(T)+O\left((\min T)^{-\varepsilon_{2}}|T|^{(n+1) / 2}\right)
$$

where $S W_{p}(T)$ is a quantity defined there so that

$$
S W_{p}(T) \gg n(T)^{-\varepsilon_{1}}|T|^{(n+1) / 2}>(\min T)^{-\varepsilon_{1}}|T|^{(n+1) / 2},
$$

and $\varepsilon_{1}, \varepsilon_{2}$ are any positive small number, and hence it gives an asymptotic formula for $r_{\mathrm{pr}}(T, S)$ when $\min T$ tends to the infinity and therefore $r_{\mathrm{pr}}(T, S)>0$ when $\min T$ is sufficiently large, and thus the above assumption on estimates of $a(T)$ yields an asymptotic formula for $r_{\mathrm{pr}}(T, S)$ and the truth of the assertion $(A)_{2 n+2, n}$. Let us refer to an asymptotic formula for the number of solutions $r(T, S)$ of $S[X]=T$. Denote by $P$ a set of primes $p$ such that the Witt index of $S$ over $\boldsymbol{Q}_{p}$ is equal to $n-1$. The assumption on $a(T)$ yields an asymptotic formula for $r(T, S)$ if $P$ is empty. Otherwise it depends on estimates of local densities from below for every prime $p \in P$ and the explicit value of $\varepsilon$ whether it gives an asymptotic formula or not. The existence of an asymptotic formula may be harmonious.

We denote by $\boldsymbol{Z}, \boldsymbol{Q}, \boldsymbol{Z}_{p}$ and $\boldsymbol{Q}_{p}$ the ring of rational integers, the field 
of rational numbers and their $p$-adic completions respectively. Terminology and notations on quadratic forms are generally those from [6] and they are also used for symmetric matrices corresponding to quadratic forms. For example, for a quadratic lattice $M$ over $Z, n M$ is the norm of $M$, i.e., $n M=Z\{Q(x) \mid x \in M\}$, and for a basis $\left\{v_{i}\right\}$ of $M$ we write $M=$ $\left\langle\left(B\left(v_{i}, v_{j}\right)\right)\right\rangle$. By a positive lattice we mean a lattice on a positive definite quadratic space over $\boldsymbol{Q}$. For a positive lattice $M, \min M$ denotes the minimum of $\{Q(x) \mid x \in M, x \neq 0\}$, where $Q(x)=B(x, x)$ is the quadratic form of $M$.

\section{$\S 1$.}

In this section we prove the following

TheOREM. Let $m, n$ be integers such that $m=2 n+2$ and $n \geq 2$ and let $M$ be a positive lattice of $\operatorname{rank} M=m$ with $n M \subset 2 Z$. Let $N$ be a positive lattice of $\operatorname{rank} N=n$ such that $Z_{p} N$ is represented by $Z_{p} M$ for each prime p. Put $n N=2 q Z$ for a natural number $q$ and decompose $q$ as $q=q_{0} q_{1}$ so that, for a prime divisor $p$ of $q, p$ divides $q_{0}$ if and only if the Witt index of $\boldsymbol{Q}_{p} M$ is equal to $n-1$. Then there exists a positive lattice $\bar{N}$ on $\boldsymbol{Q} N$ such that $\bar{N} \supset N, \min \bar{N}>c(M) \sqrt{q_{0}^{-1}} \min N$ and $Z_{p} \bar{N}$ is primitively represented by $Z_{p} M$ for each prime $p$ where $c(M)$ is a positive constant dependent only on $M$.

Corollary. If $m=2 n+2 \geq 6$, then the assertion $(A P W)_{2 n+2, n}$ yields $(A)_{2 n+2, n}$.

Before the proof of Theorem, we note that if we put $N=\langle q T\rangle$ where $T$ is an integral positive matrix, then $\min N=q(\min T)$ and hence $\min \bar{N}>c(M) \sqrt{q_{0}} q_{1} \min T$. Thus $\min \bar{N}$ is large if $\min N$ is large.

LEMMA 1. Let $a, u$ be real numbers such that $a>1$ and $\sqrt{a} / 4<u<$ $\sqrt{a}$. Put $f(x, y)=(a x-u y)^{2}+y^{2}$. Then the minimum of $\{f(x, y) \mid x, y \in Z$, $(x, y) \neq(0,0)\}$ is larger than $a / 16$.

Proof. $f(0,1)=u^{2}+1>u^{2}>a / 16$ and $f(1,0)=a^{2}>a / 16$ are clear. Suppose $x, y \in Z$ and $x y \neq 0$. If $|y|>\sqrt{ } \bar{a} / 4$, then $f(x, y) \geq y^{2}>a / 16$. Assume $|y| \leq \sqrt{a} / 4$. Since it implies $|u y|<a / 4$, the minimum of $|a x-u y|$ $(x \in Z)$ is equal to $|u y|$. Hence $f(x, y)>(a x-u y)^{2} \geq(u y)^{2} \geq u^{2}>a / 16$ holds, which completes the proof of Lemma 1 . 
Lemma 2. Let $p$ be a prime and $n \geq 2$. Let $T=p^{2 b+c} T_{0}(0<b \in Z$, $c=0,1)$ be an integral positive definite matrix of degree $n$ and suppose $p^{b} \geq 36, n T_{0} \subset 2 Z$ and $\left(n T_{0}\right) Z_{p}=2 Z_{p}$. Then there exists a positive constant $C(n, p)$ dependent on $n$ and $p$ for which there exists $H$ in $M_{n}(Z)$ satisfying that $\operatorname{det} H$ is a power of $p, \min T\left[H^{-1}\right]>C(n, p) p^{b+c} \min T_{0}, T\left[H^{-1}\right] \not \equiv 0$ $\bmod 8 p^{1+c}$ and $n\left(T\left[H^{-1}\right]\right) \subset 2 Z$.

Proof. Put $G=S L(n, Z), G^{\prime}=\left\{g \in G \mid g \equiv 1_{n} \bmod 8 p Z_{p}\right\}$, take and fix representatives $\left\{U_{i}\right\}$ of $G / G^{\prime}$ once and for all and let $C^{\prime}(n, p)$ be a positive number such that ${ }^{t} U_{i} U_{i}>C^{\prime}(n, p) 1_{n}$ for all $i$. Without loss of generality we may assume that $T_{0}$ is reduced in the sense of Minkowski and hence, as is well known, $T_{0}>C_{n}\left(\min T_{0}\right) 1_{n}$ holds for some absolute constant $C_{n}$. Since $\left(n T_{0}\right) Z_{p}=2 Z_{p}$, we can choose $V \in S L\left(n, Z_{p}\right)$ so that $T_{0}[V]=\left(\begin{array}{ll}T_{1} & 0 \\ 0 & *\end{array}\right)$ where

$$
\begin{aligned}
T_{1}= & \left(\begin{array}{cc}
2 h & 0 \\
0 & 2 k
\end{array}\right) \quad h \in Z_{p}^{\times}, k \in Z_{p}, \\
& \left(\begin{array}{cc}
2 h & k \\
k & 2 h k^{2}
\end{array}\right)=\left(\begin{array}{cc}
2 h & 1 \\
1 & 2 h
\end{array}\right)\left[\left(\begin{array}{cc}
1 & 0 \\
0 & k
\end{array}\right)\right] h=0,1, k \in Z_{p}^{\times} \quad \text { if } p=2,
\end{aligned}
$$

or

$$
\left(\begin{array}{lcc}
2 h & 0 & 0 \\
0 & 2^{i}\left(\begin{array}{cc}
2 k & 1 \\
1 & 2 k
\end{array}\right)
\end{array}\right) \quad h \in Z_{p}^{\times}, k=0,1, i \geq 2 \text { if } p=2 .
$$

Take a representative $U=U_{i}$ of $G / G^{\prime}$ so that $U \equiv V \bmod 8 p Z_{p}$; then we have $T_{0}[U]>C_{n}\left(\min T_{0}\right) 1_{n}[U]>C_{n} C^{\prime}(n, p)\left(\min T_{0}\right) 1_{n}$, and putting $A=$

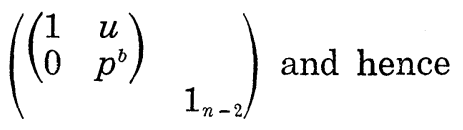

$$
p^{b} A^{-1}=\left(\begin{array}{ccc}
\left(\begin{array}{cc}
p^{b} & -u \\
0 & 1
\end{array}\right) & \\
& & p^{b} 1_{n-2}
\end{array}\right)
$$

we have

$$
\begin{aligned}
\min T\left[U A^{-1}\right] & =\min p^{2 b+c} T_{0}\left[U A^{-1}\right] \\
& >C_{n} C^{\prime}(n, p) p^{c}\left(\min T_{0}\right) \min \left(1_{n}\left[p^{b} A^{-1}\right]\right) \\
& =C_{n} C^{\prime}(n, p) p^{c}\left(\min T_{0}\right) \min \left\{\left(p^{b} x-u y\right)^{2}+y^{2}, p^{2 b}\right\}
\end{aligned}
$$

where $x, y$ run over integers not all zero, and by Lemma 1 


$$
>C_{n} C^{\prime}(n, p) p^{c}\left(\min T_{0}\right) p^{b} / 16
$$

if $\sqrt{p^{b}} / 4<u<\sqrt{p^{b}}$.

Putting $H=A U^{-1}, C(n, p)=C_{n} C^{\prime}(n, p) / 16$, we have

$$
\min T\left[H^{-1}\right]>C(n, p) p^{b+c} \min T_{0} .
$$

Since $T\left[H^{-1}\right]=p^{c} T_{0}[U]\left[p^{b} A^{-1}\right]$ and $n T_{0} \subset 2 Z$, we have $n T\left[H^{-1}\right] \subset 2 p^{c} Z$ $\subset 2 Z$. The $(2,2)$ entry of $T\left[H^{-1}\right]$ is equal $\bmod 8 p^{1+c} Z_{p}$ to

$$
2 p^{c}\left(h u^{2}+k\right), 2 p^{c}\left(h u^{2}-k u+h k^{2}\right), 2 p^{c}\left(h u^{2}+2^{i} k\right)
$$

according to the order of above canonical forms of $T_{1}$ and hence to complete the proof, it is enough to show that they are not zero modulo $8 p^{1+c}$ for some $u$ with $\sqrt{p^{b}} / 4<u<\sqrt{p^{b}}$. Noting $\sqrt{p^{b}}-\sqrt{p^{b}} / 4>4$ because of $p^{b} \geq 36$, we have only to choose $u \in Z$ with $\sqrt{p^{b}} / 4<u<\sqrt{p^{b}}$ so that $(u, p)=1$ if $k \in p Z_{p}$, and $h u^{2}+k \neq 0 \bmod p$ if $k \in Z_{p}^{\times}$in the left case; $2 \nmid u$ if $h=0$, and $2 \mid u$ if $h=1$ in the middle case: $2 \nmid u$ in the right case. Thus we have proved Lemma 2.

Remark. In the above proof, all but $(2,2)$ entries of $T\left[H^{-1}\right]$ are divided by $p^{b+c}$, and if $T_{1}$ is of the first canonical form, then $T\left[H^{-1}\right]$ represents $2 p^{c} h=p^{-2 b} \times(1,1)$ entry of $T[V]$ over $Z_{p}$ if either $p \neq 2, k \in$ $p Z_{p}$ or $p=2, k \in 8 Z_{2}$.

Proof of Theorem. First we note that for a positive lattice $K^{\prime} \supset K$, $\min K^{\prime} \geq\left[K^{\prime}: K\right]^{-2} \min K$ holds, since $\left[K^{\prime}: K\right] K^{\prime} \subset K$ implies $\min \left[K^{\prime}\right.$ : $K] K^{\prime} \geq \min K$. Let $M, N$ be those in Theorem. If a prime $p$ does not divide $d M$, then $Z_{p} M$ is unimodular and $n Z_{p} M=2 Z_{p}$. Hence $Z_{p}$ contains a submodule isometric to $\frac{1}{n}\left\langle\left(\begin{array}{ll}0 & 1 \\ 1 & 0\end{array}\right)\right\rangle$ as an orthogonal component. Therefore $Z_{p} N$ is primitively represented $Z_{p} M$. If $p \mid d M$ and ind $\boldsymbol{Q}_{p} M \geq n$, then by virtue of Theorem 2 in [4] there is an isometry $u$ from $Z_{p} N$ to $Z_{p} M$ such that $\left[\boldsymbol{Q}_{p} u\left(Z_{p} N\right) \cap Z_{p} M_{p}: u\left(Z_{p} N\right)\right]$ is bounded by a number $C_{p}$ dependent only on $Z_{p} M$. Hence $\bar{N}_{p}=u^{-1}\left(\boldsymbol{Q}_{p} u\left(Z_{p} N\right) \cap Z_{p} M\right)\left(\supset Z_{p} N\right)$ is primitively represented by $Z_{p} M$, and enlarging $N$ to $N^{\prime \prime}$ so that $Z_{p} N^{\prime \prime}=$ $\bar{N}_{p}, Z_{p} N^{\prime \prime}$ is primitively represented by $Z_{p} M$ and $\min N^{\prime \prime} \geq C_{p}^{-2} \min N$. Suppose that $p \mid d M$ and ind $\boldsymbol{Q}_{p} M=n-1$. We fix a $2 p^{k_{p}} \boldsymbol{Z}_{p}$-maximal sublattice $K$ of $Z_{p} M$ for some $k_{p}$ once and for all. If $n Z_{p} N \supset 2 p^{2+k_{p}} Z_{p}$, then there is an isometry $u$ from $Z_{p} N$ to $Z_{p} M$ such that $\left[Q_{p} u\left(Z_{p} N\right) \cap\right.$ $\left.Z_{p} M: u\left(Z_{p} N\right)\right]$ is bounded by a number $C_{p}$ dependent only on $k_{p}$ and $Z_{p} M$, applying the theorem referred above where $N_{1}$ there, should be the first Jordan component of $Z_{p} N$, and nothing that the number of distinct 
isometry classes by $O\left(Z_{p} M\right)$ of modular submodules of $Z_{p} M$ with $n \supset$ $2 p^{2+k_{p}} Z_{p}$ is finite. In this case we have obtained an enlarged quadratic lattice of $N$ at $p$ which contains $N$ with index dependent only on $k_{p}$ and $Z_{p} M$ and is primitively represented by $M$ over $Z_{p}$. Finally we deal with the case that $p \mid d M$, ind $\boldsymbol{Q}_{p} M=n-1$ and $n \boldsymbol{Z}_{p} N \subset 2 p^{2+k_{p}} \boldsymbol{Z}_{p}$. Put $N=\left\langle p^{2 b+c+k_{p}} T_{0}\right\rangle$ where $0<b \in Z, c=0,1$ and $n T_{0} \subset 2 Z,\left(n T_{0}\right) Z_{p}=2 Z_{p}$. By virtue of Lemma 2 , there exists a matrix $H$ in $M_{n}(Z)$ such that $\operatorname{det} H$ is a power of $p$,

$$
\begin{aligned}
\min p^{2 b+c} T_{0}\left[H^{-1}\right] & >C(n, p) p^{b+c} \min T_{0}, \\
p^{2 b+c} T_{0}\left[H^{-1}\right] & \neq 0 \bmod 8 p^{1+c} \text { and } n\left(p^{2 b+c} T_{0}\left[H^{-1}\right]\right) \subset 2 Z .
\end{aligned}
$$

Taking a quadratic lattice $N^{\prime}(\supset N)$ corresponding to $H, N^{\prime}$ satisfies $n\left(Z_{p} N^{\prime}\right) \subset 2 p^{k_{p}} \boldsymbol{Z}_{p}=n K, \quad n\left(\boldsymbol{Z}_{p} N^{\prime}\right) \not \subset 8 p^{1+c+k_{p}} \boldsymbol{Z}_{p}$ and $\min N^{\prime}>C(n, p) p^{b+c+k_{p}}$ $\min T_{0} \geq C(n, p) p^{\left(2 b+c+k_{p}\right) / 2} \min T_{0}=C(n, p) p^{-\left(\operatorname{ord}_{p} q_{0}\right) / 2} \min N$. Since $\boldsymbol{Q}_{p} N^{\prime}=$ $\boldsymbol{Q}_{p} N$ is represented by $\boldsymbol{Q}_{p} M=\boldsymbol{Q}_{p} K, Z_{p} N^{\prime}$ is represented by the maximal lattice $K$ and hence by $Z_{p} M$. Applying the argument in the case of $p \mid 2 d M, n Z_{p} N \supset 2 p^{2+k_{p}} Z_{p}$ to $N^{\prime}, M$, noting $n\left(Z_{p} N^{\prime}\right) \not \subset 8 p^{1+c+k_{p}} Z_{p}$, there is a lattice $N^{\prime \prime}\left(\supset N^{\prime}\right)$ such that $\left[N^{\prime \prime}: N^{\prime}\right]$ is a power of $p$ bounded by a number dependent on $k_{p}$ and $Z_{p} M$, and $Z_{p} N^{\prime \prime}$ is primitively represented by $Z_{p} M$. Iterating the construction of $N^{\prime \prime}$ for primes $p$ dividing $d M$, we complete the proof of Theorem.

Remark. Let us consider the case $m=2 n+1$. Let $M$ be a positive lattice of $\mathrm{rk} M=m$ and $N$ a positive lattice of $\mathrm{rk} N=n$ which is represented by gen $M$. It is easy to see that the assertion similar to Theorem holds, using Lemma 2 and its remark, provided that for every prime $p$ for which ind $\boldsymbol{Q}_{p} M=n-1$ holds and $Z_{p} N$ has a Jordan splitting $Z_{p} N=$ $\langle a\rangle \perp N_{1}$ where $\operatorname{ord}_{p} a$ is bounded but $\operatorname{ord}_{p} n N_{1}$ is large, there is a lattice $\bar{N}$ such that $[\bar{N}: N]$ is a power of $p, Z_{p} \bar{N}$ is represented by $Z_{p} M, Z_{p} \bar{N}$ contains a binary lattice $B$ with $\operatorname{ord}_{p} d B$ bounded and $\min \bar{N}$ is larga.

This condition is not necessarily satisfied for $n=2$ as follows: For $N=\langle a\rangle \perp\left\langle p^{r}\right\rangle$ with $(a, p)=1, \bar{N}=\langle a\rangle \perp\left\langle p^{r-2 t}\right\rangle$ holds if $[\bar{N}: N]=p^{t}$. Thus $\min \bar{N}$ is small if $\operatorname{ord}_{p} \bar{N}$ is small. This leads us to a falsehood of the assertion $(A)_{m, n}$ when $m=2 n+1=n+3, n=2$, as in [2].

$\S 2$.

We have observed that it is important whether for a given sequence $\left\{N_{t}\right\}$ of positive lattices represented by gen $M$ with $\min N_{t} \rightarrow \infty$, there is 
a lattice $\bar{N}_{t}$ with min $\bar{N}_{t}$ large which contains $N_{t}$ and is primitively represented at every spot by gen $M$ or not. If there is no such $\bar{N}_{t}$, then we must deduce a falsehood of the assertion $(A)_{m, n}$.

In this section we show that it is hard to construct such a sequence by scalings of a fixed lattice by giving the following

Proposition. Let $M, N$ be positive lattices of $\operatorname{rk} M=m \geq \operatorname{rk} N+3$, $\operatorname{rk} N=n \geq 3$. We fix representatives $\left\{N_{i}\right\}$ of classes in the genus of $N$ once and for all, and take a finite set $S$ ( $\ni 2)$ of primes such that if $p \notin S$, then $Z_{p} N_{i}=Z_{p} N$ holds for all $i$ and $Z_{p} M, Z_{p} N$ are unimodular. For any given number $C_{1}$, there is a positive number $C_{2}=C_{2}\left(C_{1}, M, N\right)$ such that if a natural number $a\left(\geq C_{2}\right)$ is not divided by any prime in $S$ and the scaling $N(a)$ of $N$ by $a$ is locally represented by $M$, then there is a lattice $\bar{N}_{a}$ with $\min \bar{N}_{a} \geq C_{1}$ which contains $N(a)$ and $Z_{p} \bar{N}_{a}$ is primitively represented by $Z_{p} M$ for every prime $p$.

Corollary. For the above special sequence $\{N(a)\}$, the assertion $(A P W)_{m, n}$ implies the assertion $(A)_{m, n}$.

This follows trivially and to prove Proposition, we must prepare the following

TheOREm. Let $L$ be a positive lattice of $n L=2 Z$ and $\operatorname{rk} L=m \geq 2$. For a prime $p$ we define an integer $a_{p}$ by the following:

If $m \geq 3$ and the Jordan splitting is of form

$$
Z_{p} L=\left\langle 2 \varepsilon_{1}\right\rangle \perp\left\langle 2 \varepsilon_{2} p^{a_{p}}\right\rangle \perp \cdots \quad p \geq 2,
$$

or

$$
\left\langle 2 \varepsilon_{1}\right\rangle \perp\left\langle 2^{a_{2}}\left(\begin{array}{cc}
2 c & 1 \\
1 & 2 c
\end{array}\right)\right\rangle \perp \cdots \quad p=2,
$$

where $\varepsilon_{1}, \varepsilon_{2} \in \boldsymbol{Z}_{p}^{\times}$and $c=0$ or 1 , then $a_{p}$ is given as in the above, otherwise $a_{p}=0$. Then there is a lattice $M$ in the genus of $L$ such that

$$
\min M \gg(d L)^{1 / m-\varepsilon}\left(\prod_{p \mid 2 d L} p^{a_{p}}\right)^{-1 / m}
$$

where $\varepsilon$ is any positive number and $A \gg B$ means $A>c B$ for a constant $c$ dependent only on $\varepsilon$ and $m$.

Remark. $\min L \ll(d L)^{1 / m}$ is well known.

Before the proof of Theorem we show that Proposition follows from Theorem. 
Let $M, N, N_{i}, S$ be those in Proposition. For a prime $p$, let $K=Z_{p}[e, f]$ be a quadratic lattice over $Z_{p}$ defined by $Q(e)=Q(f)=0, B(e, f)=a$. Then $\bar{K}=Z_{p}\left[a^{-1} e, f\right]=\left\langle\left(\begin{array}{ll}0 & 1 \\ 1 & 0\end{array}\right)\right\rangle$ is clear. Hence for a prime $p$ dividing $a$ we can take a lattice $\bar{N}_{p}$ which contains $Z_{p} N(a)$ and is isometric to an orthogonal sum of a unimodular lattice of $\mathrm{rk}=n-1$ or $n-2$ and an $a Z_{p}$-modular lattice of $\mathrm{rk}=1$ or 2 , enlarging binary hyperbolic $a Z_{p^{-}}$ modular lattices to unimodular lattices as above. Let $N^{\prime}$ be a lattice which is isometric to $\bar{N}_{p}$ for $p \mid a$ and to $Z_{p} N(a)$ for $p \nmid a$ and has a large minimum by virtue of Theorem. Since there is an isometry from $Z_{p} N(a)$ to $Z_{p} N^{\prime}$ for every prime and $\boldsymbol{Q} N(a)=\boldsymbol{Q} N^{\prime}, N^{\prime}$ contains a lattice which is isometric to $N_{i}(a)$ for some $i$. Pulling back $N^{\prime}$, there is a lattice $N^{\prime \prime}$ such that $\min N^{\prime \prime}$ is large, $N^{\prime \prime} \supset N_{i}(a), Z_{p} N^{\prime \prime}=Z_{p} N_{i}(a)$ for $p \nmid a$ and $Z_{p} N^{\prime \prime}$ has a unimodular component of $\mathrm{rk}=n-1$ or $n-2$ for $p \mid a$. Define a new lattice $\bar{N}$ by $Z_{p} \bar{N}=Z_{p} N(a)$ for $p \nmid a$ and $Z_{p} \bar{N}=Z_{p} N^{\prime \prime}$ for $p \mid a$. By definition $\bar{N}$ contains $N(a)$ and $Z_{p} \bar{N}=Z_{p} N^{\prime \prime}$ if $p \notin S$ and $p \nmid a$. Since

$$
\begin{aligned}
{\left[\bar{N}: \bar{N} \cap N^{\prime \prime}\right] } & =\prod\left[Z_{p} \bar{N}: Z_{p} \bar{N} \cap Z_{p} N^{\prime \prime}\right]=\prod_{p \in S}\left[Z_{p} \bar{N}: Z_{p} \bar{N} \cap Z_{p} N^{\prime \prime}\right] \\
& =\prod_{p \in S}\left[Z_{p} N(a): Z_{p} N(a) \cap Z_{p} N_{i}(a)\right]=\left[N: N \cap N_{i}\right]
\end{aligned}
$$

and $\left[\bar{N}: \bar{N} \cap N^{\prime \prime}\right]^{2} \min \bar{N} \geq \min \left(\bar{N} \cap N^{\prime \prime}\right)$, we have $\min \bar{N} \geq\left[N: N \cap N_{i}\right]^{-2}$ $\times \min \left(\bar{N} \cap N^{\prime \prime}\right) \geq\left[N: N \cap N_{2}\right]^{-2} \min N^{\prime \prime}$. Thus we have constructed a lattice $\bar{N}$ which contains $N(a)$, has a large minimum and satisfies that $Z_{p} \bar{N}=Z_{p} N(a)$ for $p \nmid a$ and $Z_{p} \bar{N}$ has a unimodular component of $\mathrm{rk}=n-1$ or $n-2$ for $p \mid a$. By assumption, $N(a)$ is represented by $M$ locally and $Z_{p} N, Z_{p} M$ are unimodular if $p \notin S$. Hence $Z_{p} \bar{N}$ is primitively represented by $Z_{p} M$ if $p \notin S$ and $p \nmid a$. If $p \mid a$, then by cancellation of a unimodular component of $Z_{p} N$ from $Z_{p} \bar{N}$ and $Z_{p} M$, the remaining part of $Z_{p} \bar{N}$ is primitively represented by the one of $Z_{p} M$ and hence $Z_{p} \bar{N}$ is primitively represented by $Z_{p} M$. Enlarging $\bar{N}$ for every prime $p \in S$ we get a lattice $\bar{N}_{a}$ which contains $N(a)$, is primitively represented by $M$ locally and has a large minimum since $\left[\bar{N}_{a}: \bar{N}\right]=\prod_{p \in S}\left[Z_{p} \bar{N}_{a}: Z_{p} N(a)\right]$ is bounded by a number depending on $N$ and $M$. Thus we have completed the proof of Proposition, assuming Theorem.

Proof of Theorem. We divide the proof to two cases $m=2$ and $m \geq 3$. First we treat the case $m=2$.

Lemma. For given natural numbers $a$ and $D$, the number of $b, c$ 
which satisfy $0 \leq b \leq a \leq c$ and $D=4 a c-b^{2}$, is $O\left(a^{\varepsilon}(D, a)^{1 / 2}\right)$ where $\varepsilon$ is any positive number.

Proof. The number of $b, c$ is less than or equal to $\sharp\left\{b \bmod 4 a \mid b^{2} \equiv\right.$ $-D \bmod 4 a\}$. First we show, for a prime power $p^{n}$, $\sharp\left\{x \bmod p^{n} \mid x^{2} \equiv-\right.$ $\left.D \bmod p^{n}\right\} \leq 4\left(D, p^{n}\right)^{1 / 2}$. Put $d=\operatorname{ord}_{p} D$. If $d \geq n$, then $\sharp\left\{x \bmod p^{n} \mid x^{2} \equiv\right.$ $\left.-D \bmod p^{n}\right\}=\#\left\{x \bmod p^{n} \mid x^{2} \equiv 0 \bmod p^{n}\right\}=p^{[n / 2]}<4\left(D, p^{n}\right)^{1 / 2}$ holds, where [r] means the largest integer which does not exceed $r$. Suppose $d<n$. If $x^{2} \equiv-D \bmod p^{n}$, then $d$ is even and $x=p^{d / 2} y$ for an integer $y$ satisfying $y^{2} \equiv-D p^{-d} \bmod p^{n-d}$. The number of solutions modulo $p^{n-d}$ for $y^{2} \equiv-D p^{-d} \bmod p^{n-d}$ is at most four, and for each $y, x=p^{d / 2}\left(y+p^{n-d} z\right)$ $\left(z \bmod p^{d / 2}\right)$ is a solution. This completes the above inequality. Hence $\#\left\{b \bmod 4 a \mid b^{2} \equiv-D \bmod 4 a\right\} \leq\left(\prod_{p \mid 4 a} 4\right)(D, 4 a)^{1 / 2} \ll a^{\varepsilon}(D, a)^{1 / 2}$.

Let $L$ be a binary positive lattice with $n L=2 Z, d L=D$, and denote by $h$ the number of isometry classes in gen $L$. Every binary even positive lattice corresponds to the only one reduced matrix $\left(\begin{array}{cc}2 a & b \\ b & 2 c\end{array}\right) 0 \leq b$ $\leq a \leq c$. Hence we have

$$
\begin{aligned}
& \sum_{a=1}^{k} \#\{M \in \text { gen } L / \mathrm{cls} \mid \min M=2 a\} \\
\ll & \sum_{a=1}^{k} a^{\varepsilon}(D, a)^{1 / 2} \\
\ll & \sum_{s \mid D} \sum_{1 \leq t \leq k / s}(s t)^{\varepsilon} s^{1 / 2} \\
\ll & \sum_{s \mid D} s^{1 / 2+\varepsilon}(k / s)^{1+\varepsilon} \\
= & k^{1+\varepsilon} \sum_{s \mid D} s^{-1 / 2} \ll k^{1+\varepsilon} D^{\varepsilon} .
\end{aligned}
$$

Thus there is a number $c$ dependent only on $\varepsilon$ so that

$$
\sum_{a=1}^{k} \#\{M \in \text { gen } L / \operatorname{cls} \mid \min M=2 a\}<c k^{1+\varepsilon} D^{\varepsilon} .
$$

If the class number $h$ of gen $L$ is greater than $c k^{1+\varepsilon} D^{\varepsilon}$, then there is a lattice $M \in$ gen $L$ such that $\min M>k$. By Siegel, $h \gg D^{1 / 2-\varepsilon}$ is well known. Noting that $\varepsilon$ 's are any positive numbers, we have $\min M \gg$ $D^{1 / 2-\varepsilon}$ for any $\varepsilon>0$, which completes the proof in the case $m=2$.

To treat the case $m \geq 3$, we prepare several lemmas. Let us denote by $p$ a prime number.

LemMa 1. Let $a$ and $b$ be integers and $a \geq b \geq 0$. For $\alpha \in Z_{p}$ with 
$\operatorname{ord}_{p} \alpha=b$, the number $t$ of solutions modulo $p^{a}$ of $x^{2} \equiv \alpha \bmod p^{a}$ is $O\left(p^{b / 2}\right)$.

Proof. Suppose $a=b$; then $t$ is equal to $\sharp\left\{x \bmod p^{a} \mid x^{2} \equiv 0 \bmod p^{a}\right\}$ $=p^{[a / 2]} \leq p^{b / 2}$. Suppose $a>b$. If $b$ is odd, then there is no solution and hence $t=0$. If $b$ is even and $b=2 d$, then $t$ is equal to

$$
\begin{aligned}
& \#\left\{y \bmod p^{a-d} \mid y^{2} \equiv \alpha p^{-2 d} \bmod p^{a-2 d}\right\} \\
= & p^{d} \#\left\{y \bmod p^{a-2 d} \mid y^{2} \equiv \alpha p^{-2 d} \bmod p^{a-2 d}\right\} \\
\leq & 4 p^{d}=O\left(p^{b / 2}\right) .
\end{aligned}
$$

LEMMA 2. For $0 \leq a \leq h-1, \varepsilon \in Z_{p}^{\times}$and $\alpha \in Z_{p}$, we put $t=\#\left\{x \bmod p^{h}\right.$, $\left.y \bmod p^{h-a} \mid x^{2}+\varepsilon p^{a} y^{2} \equiv \alpha \bmod p^{h},(x, y)=1\right\}$. Then $t=O\left(p^{h-a / 2}\right)$ holds.

Proof. Let $t_{1}$ (resp. $t_{2}$ ) be the number of solutions under an additional condition $p \mid y$ (resp. $p \nmid y$ ). $t=t_{1}+t_{2}$ is clear. Without loss of generality we may put $\alpha=\delta p^{c}, \delta \in Z_{p}^{\times}, 0 \leq c \leq h$. Then $t_{1}$ is equal to

$$
\#\left\{x \bmod p^{h}, y \bmod p^{h-a-1} \mid x^{2}+\varepsilon p^{a+2} y^{2} \equiv \alpha \bmod p^{h}, p \nmid x\right\} .
$$

If $c>0$ i.e., $p \mid \alpha$, then $t_{1}=0$ holds. If $c=0$, then $\alpha-\varepsilon p^{a+2} y^{2}$ is in $Z_{p}^{\times}$ and hence $t_{1}=O\left(p^{h-a-1}\right)=O\left(p^{h-a / 2}\right) . \quad t_{2}$ is equal to

$$
\begin{aligned}
& \sum_{\substack{x \bmod p^{h} \\
x^{2} \equiv \delta p^{c} \bmod p^{a}}} \sharp\left\{y \bmod p^{h-a} \mid \varepsilon p^{a} y^{2} \equiv \delta p^{c}-x^{2} \bmod p^{h}, p \nmid y\right\} . \\
& =\sum_{\substack{x \bmod \\
x^{2} \equiv \dot{\rho} p^{c} \bmod p^{a}}} \sharp\left\{y \bmod p^{h-a} \mid y^{2} \equiv\left(\varepsilon p^{a}\right)^{-1}\left(\delta p^{c}-x^{2}\right) \bmod p^{h-a}, p \nmid y\right\} \\
& \ll \#\left\{x \bmod p^{h} . \operatorname{ord}_{p}\left(x^{2}-\delta p^{c}\right)=a\right\} .
\end{aligned}
$$

We show that this is $O\left(p^{h-a / 2}\right)$ in each case of $c \geq a, c<a$. Suppose $c \geq a$; then $t_{2} \ll \#\left\{x \bmod p^{h} \mid x^{2} \equiv 0 \bmod p^{a}\right\}=p^{h-[(a+1) / 2]} \leq p^{h-a / 2}$. Suppose $c<a$. If $x^{2}-\delta p^{c} \equiv 0 \bmod p^{a}$ is soluble, then $2 \mid c$ and $x=p^{c / 2} z$ for some $z \in Z_{p}$. Hence $t_{2}$ is less than

$$
\begin{aligned}
& \#\left\{z \bmod p^{h-c / 2} \mid \operatorname{ord}_{p}\left(p^{c}\left(z^{2}-\delta\right)\right)=a\right\} \\
\leq & \#\left\{z \bmod p^{h-c / 2} \mid z^{2} \equiv \delta \bmod p^{a-c}\right\} \\
= & p^{h-c / 2-(a-c)} \#\left\{z \bmod p^{a-c} \mid z^{2} \equiv \delta \bmod p^{a-c}\right\} \\
= & O\left(p^{h-a+c / 2}\right)=O\left(p^{h-a / 2}\right) .
\end{aligned}
$$

Thus we have completed the proof.

Lemma 3. For integers $a, c$ and $h$ satisfying $0 \leq a \leq h-1$ and $0 \leq$ $c \leq h$ and for $\varepsilon, \delta \in Z_{p}^{\times}$, we put 


$$
t=\#\left\{x \bmod p^{h}, y \bmod p^{h-a} \mid x^{2}+\varepsilon p^{a} y^{2} \equiv \delta p^{c} \bmod p^{h}\right\} .
$$

Then we have $t=O\left(h p^{h-a / 2}\right)$.

Proof. $t$ is equal to

$$
\begin{aligned}
& \sum_{0 \leq i \leq h-a} \#\left\{x \bmod p^{h}, y \bmod p^{h-a} \mid x^{2}+\varepsilon p^{a} y^{2} \equiv \delta p^{c} \bmod p^{h},(x, y)=p^{i}\right\} \\
= & t_{1}+t_{2}+t_{3},
\end{aligned}
$$

where $t_{1}, t_{2}$ and $t_{3}$ are partial sums under conditions $2 i<c, 2 i=c$ and $2 i>c$ respectively. Further we divide $t_{1}$ to the sum of $t_{1,1}$ and $t_{1,2}$ where $t_{1,1}, t_{1,2}$ are partial sums under conditions $i<(h-a) / 2, i \geq(h-a) / 2$ respectively. $t_{1,1}$ is equal to

$\sum_{\substack{0 \leq i<(<-a) / 2 \\ i<c / 2}} \sharp\left\{x \bmod p^{h-i}, y \bmod p^{h-a-i} \mid x^{2}+\varepsilon p^{a} y^{2} \equiv \delta p^{c-2 i} \bmod p^{h-2 i},(x, y)=1\right\}$

and considering $x \bmod p^{h-2 i}, y \bmod p^{h-a-2 i}$ and using Lemma 2 we have $t_{1,1} \ll \sum_{\substack{0 \leq i<(h-a) / 2 \\ i<c / 2}} p^{2 i+(h-2 i-a / 2)}<h p^{h-a / 2} . \quad t_{1,2}$ is equal to

$$
\begin{gathered}
\sum_{(h-a)) 2 \leq i \leq h-a} \sharp\left\{x \bmod p^{h-i}, y \bmod p^{h-a-i} \mid x^{2}+\varepsilon p^{a} y^{2} \equiv \delta p^{c-2 i} \bmod p^{h-2 i},\right. \\
\leq \sum_{(h-a) / 2 \leq i<c / 2} \sharp\left\{x \bmod p^{h-i}, y \bmod p^{h-a-i} \mid x^{2} \equiv \delta p^{c-2 i} \bmod p^{h-2 i},(x, y)=1\right\}
\end{gathered}
$$

because of $h-2 i \leq a$,

$$
\begin{aligned}
& <\sum_{(h-a) / 2 \leq i<c / 2} p^{h-a-i} \#\left\{x \bmod p^{h-i} \mid x^{2} \equiv \delta p^{c-2 i} \bmod p^{h-2 i}\right\} \\
& =\sum_{(h-a) / 2 \leq i<c / 2} p^{h-a} \#\left\{x \bmod p^{h-2 i} \mid x^{2} \equiv \delta p^{c-2 i} \bmod p^{h-2 i}\right\} \\
& \ll p^{h-a} \sum_{(h-a) / 2 \leq i<c / 2} p^{(c-2 i) / 2} \quad(\text { by Lemma 1) } \\
& <p^{h-a+c / 2} \sum_{(h-a) / 2 \leq i} p^{-i} \\
& \ll p^{h-a+c / 2-(h-a) / 2} \leq p^{h-a / 2} .
\end{aligned}
$$

Since $t_{2}$ is zero if $2 \nmid c$, we may assume $2 / c$ and hence we have $0 \leq c / 2$ $\leq h-a . \quad t_{2}$ is equal to

$$
\begin{gathered}
\#\left\{x \bmod p^{h-c / 2}, y \bmod p^{h-a-c / 2} \mid x^{2}+\varepsilon p^{a} y^{2} \equiv \delta \bmod p^{h-c},(x, y)=1\right\} \\
=p^{c / 2} \#\left\{x \bmod p^{h-c}, y \bmod p^{h-a-c / 2} \mid x^{2}+\varepsilon p^{a} y^{2} \equiv \delta \bmod p^{h-c},(x, y)=1\right\} .
\end{gathered}
$$

If $a=0$, then $t_{2}$ is equal to

$$
\begin{aligned}
& p^{c} \#\left\{x, y \bmod p^{h-c} \mid x^{2}+\varepsilon y^{2} \equiv \delta \bmod p^{h-c},(x, y)=1\right\} \\
& \ll p^{h} \quad \text { (by Lemma 2) }=p^{h-a / 2} .
\end{aligned}
$$


If $a>0$, then $t_{2}$ is less than or equal to

$$
\begin{aligned}
& p^{c / 2} \sum_{y \bmod } \sum_{p^{h-a-c / 2}} \#\left\{x \bmod p^{h-c} \mid x^{2} \equiv \delta-\varepsilon p^{a} y^{2} \bmod p^{h-c}\right\} \\
& \left.\ll p^{c / 2+h-a-c / 2} \quad \text { (by Lemma } 1\right) \\
& <p^{n-a / 2} \text {. }
\end{aligned}
$$

If $c<h$, then $t_{3}$ is equal to 0 , and hence we may put $c=h$. Then $t_{3}$ is equal to

$$
\begin{aligned}
& \sum_{h / 2<i \leq h-a} \sharp\left\{x \bmod p^{h}, y \bmod p^{h-a} \mid(x, y)=p^{i}\right\} \\
< & \sum_{i>h / 2} p^{(h-i)+(h-a-i)} \ll p^{2 h-a-h}<p^{h-a / 2} .
\end{aligned}
$$

Summing up, we complete the proof.

Lemma 4. Put $t=\#\left\{x, y \bmod 2^{h} \mid x y \equiv a \bmod 2^{h}\right\}$ for an integer $a$. Then $t \ll h \cdot 2^{h}$ holds.

Proof. $t$ is equal to

$$
\begin{aligned}
& \sum_{0 \leq i \leq h} \#\left\{x \bmod 2^{h-i}, y \bmod 2^{h} \mid 2^{i} x y \equiv a \bmod 2^{h}, 2 \nmid x\right\} \\
= & \sum_{0 \leq i \leq h} \varphi\left(2^{h-i}\right) \#\left\{y \bmod 2^{h} \mid 2^{i} y \equiv a \bmod 2^{h}\right\},
\end{aligned}
$$

where $\varphi$ means the Euler's function

$$
<\sum_{0 \leq i \leq h} 2^{h-i} \cdot 2^{i} \leq(h+1) 2^{h} \ll h \cdot 2^{h} .
$$

Lemma 5. Put $t=\sharp\left\{x, y \bmod 2^{h} \mid x^{2}+x y+y^{2} \equiv a \bmod 2^{h}\right\}$ for an integer a. Then $t \ll 2^{h}$ holds.

Proof. Put $a=b \cdot 2^{c}, 2 \nmid b$, and note that $x^{2}+x y+y^{2} \equiv 0 \bmod 2^{n}$ implies $x^{2} \equiv y^{2} \equiv 0 \bmod 2^{n}$. If $c \geq h$, then $t$ is equal to

$$
\begin{aligned}
& \#\left\{x, y \bmod 2^{h} \mid x^{2}+x y+y^{2} \equiv 0 \bmod 2^{h}\right\} \\
\leq & \#\left\{x, y \bmod 2^{h} \mid x^{2} \equiv y^{2} \equiv 0 \bmod 2^{h}\right\} \\
\ll & 2^{h} .
\end{aligned}
$$

If $c<h$ and $2 \nmid c$, then we have $t=0$. Suppose $c<h$ and $2 \mid c$; then $t$ is equal to

$$
\begin{aligned}
& \#\left\{x, y \bmod 2^{h-c / 2} \mid x^{2}+x y+y^{2} \equiv b \bmod 2^{h-c}\right\} \\
= & 2^{c} \#\left\{x, y \bmod 2^{h-c} \mid x^{2}+x y+y^{2} \equiv b \bmod 2^{h-c}\right\} \\
\leq & 2^{c+1} \#\left\{x, y \bmod 2^{h-c} \mid x^{2}+x y+y^{2} \equiv b \bmod 2^{h-c}, 2 \nmid y\right\} .
\end{aligned}
$$


Here we claim that there is at most 2 solutions of $x$ for $x^{2}+x y+y^{2}$ $\equiv b \bmod 2^{h-c}$ for an odd $y$. Suppose that $x_{1}, x_{2}$ are solutions. Then $\left(x_{1}-x_{2}\right)\left(x_{1}+x_{2}+y\right) \equiv 0 \bmod 2^{h-c}$ holds. Since only one of $x_{1}-x_{2}, x_{1}+$ $x_{2}+y$ is odd, only one of $x_{1}-x_{2} \equiv 0 \bmod 2^{h-c}$ or $x_{1}+x_{2}+y \equiv 0 \bmod 2^{h-c}$ can occur, and hence the number of solutions is at most 2. Thus $t \leq$ $2^{c+2} \varphi\left(2^{h-c}\right) \ll 2^{h}$ holds.

LeMma 6. For $h>a \geq 1 p u t$

$$
t=\#\left\{x \bmod 2^{h-1}, y, z \bmod 2^{h-a} \mid 2 x^{2}+2^{a+1} y z \equiv b \bmod 2^{h+1}\right\}
$$

for an integer $b$. Then $t \ll h \cdot 2^{2 h-3 a / 2}$ holds.

Proof. If $b$ is odd, then $t$ is clearly zero, and hence we may put $b=d \cdot 2^{c+1}, 2 \nmid d, c \geq 0$. Then $t$ is equal to

$$
\begin{aligned}
& \sum_{x \bmod 2^{h-1}} \#\left\{y, z \bmod 2^{h-a} \mid 2^{a} y z \equiv d \cdot 2^{c}-x^{2} \bmod 2^{h}\right\} \\
= & \sum_{\substack{x \bmod 2^{h-1} \\
x^{2} \equiv d \cdot 2^{c} \bmod 2^{a}}} \#\left\{y, z \bmod 2^{h-a} \mid y z \equiv 2^{-a}\left(d \cdot 2^{c}-x^{2}\right) \bmod 2^{h-a}\right\} \\
\ll & (h-a) 2^{h-a} \#\left\{x \bmod 2^{h-1} \mid x^{2} \equiv d \cdot 2^{c} \bmod 2^{a}\right\} \quad \text { (by Lemma 4) } \\
< & h \cdot 2^{2(h-a)} \#\left\{x \bmod 2^{a} \mid x^{2} \equiv d \cdot 2^{c} \bmod 2^{a}\right\} \\
\ll & h \cdot 2^{2(h-a)+\min (c, a) / 2} \quad(\text { by Lemma } 1) \\
< & h \cdot 2^{2 h-3 a / 2} .
\end{aligned}
$$

Lemma 7. For $h>a \geq 1$ put

$t=\#\left\{x \bmod 2^{h-1}, y, z \bmod 2^{h-a} \mid 2 x^{2}+2^{a+1}\left(y^{2}+y z+z^{2}\right) \equiv b \bmod 2^{h+1}\right\}$.

Then we have $t \ll 2^{2 h-3 a / 2}$.

Proof. Put $b=d \cdot 2^{c+1}, 2 \nmid d, c \geq 0$; then $t$ is equal to

$$
\begin{aligned}
& \sum_{\substack{x \bmod 2^{h-1} \\
x^{2} \equiv d \cdot 2^{c} \bmod 2^{a}}} \#\left\{y, z \bmod 2^{h-a} \mid y^{2}+y z+z^{2} \equiv 2^{-a}\left(d \cdot 2^{c}-x^{2}\right) \bmod 2^{h-a}\right\} \\
\ll & 2^{h-a} \#\left\{x \bmod 2^{h-1} \mid x^{2} \equiv d \cdot 2^{c} \bmod 2^{a}\right\} \quad(\text { by Lemma } 5) \\
\ll & 2^{2(h-a)} \#\left\{x \bmod 2^{a} \mid x^{2} \equiv d \cdot 2^{c} \bmod 2^{a}\right\} \\
\ll & 2^{2 h-3 a / 2}
\end{aligned}
$$

as in the proof of Lemma 6.

Recall that $L$ is a positive lattice of $n L=2 Z, \operatorname{rk} L=m \geq 3$.

LEMMA 8. We have $\prod_{p \nmid 2 d L} \alpha_{p}(t, L) \ll(t d L)^{\varepsilon}$ for a natural number $t$ and any positive number $\varepsilon$ where $\alpha_{p}$ is the local density. 
Proof. For a prime number $p$ not dividing $2 d L$ we put $\delta=\delta_{p}=$ $\chi\left((-1)^{m / 2} d L\right)$ (resp. $\chi\left((-1)^{(m-1) / 2} t p^{-e} d L\right), r=r_{p}=p^{1-m / 2}$ (resp. $p^{2-m}$ ) for $2 \mid m$ (resp. $2 \nmid m$ ), where $\chi$ is the quadratic residue symbol for $p$ and $e=e_{p}=$ $\operatorname{ord}_{p} t$.

By Hilfssatz 16 in [9], $\alpha_{p}(t, L)$ is equal to

$$
\begin{array}{ll}
\left(1-\delta p^{-m / 2}\right)\left(1+\delta r+\cdots+(\delta r)^{e}\right) & 2 \mid m, \\
\left(1-p^{1-m}\right)\left(1+r+\cdots+r^{(e-1) / 2}\right) & 2 \nmid e, 2 \nmid m, \\
\left(1-p^{1-m}\right)\left\{1+r+\cdots+r^{e / 2-1}+r^{e / 2}\left(1-\delta p^{(1-m) / 2}\right)^{-1}\right\} & 2 \mid e, 2 \nmid m .
\end{array}
$$

If $m$ is even, then we have

$$
\begin{aligned}
\alpha_{p}(t, L) & \leq\left(1+p^{-m / 2}\right) \sum_{k \geq 0} r^{k} \\
& =\left(1+p^{-m / 2}\right)\left(1-p^{1-m / 2}\right)^{-1} .
\end{aligned}
$$

Hence for an even integer $m(\geq 3)$ we have

$$
\begin{aligned}
\prod_{p \nmid 2 d L} \alpha_{p}(t, L) & <\prod_{p \nmid 2 d L}\left(1+p^{-m / 2}\right) \prod_{p \mid t}\left(1-p^{1-m / 2}\right)^{-1} \\
& \ll \prod_{p \mid t}\left(1-p^{1-m / 2}\right)^{-1} \leq \prod_{p \mid t}\left(1-p^{-1}\right)^{-1} \ll t^{\varepsilon}
\end{aligned}
$$

for any positive $\varepsilon$, since $\varphi(t)>c t(\log \log t)^{-1}$ for $t \geq 3$ and some positive number $c$.

Suppose $2 \nmid m$. If $2 \nmid e$, then we have

$$
\begin{aligned}
\alpha_{p}(t, L) & =\left(1-p^{1-m}\right)\left(1-p^{(2-m)(e+1) / 2}\right)\left(1-p^{2-m}\right)^{-1} \\
& <\left(1-p^{2-m}\right)^{-1}<\left(1-p^{2-m}\right)^{-1}\left(1-p^{(1-m) / 2}\right)^{-1}
\end{aligned}
$$

If $e=0$, then we have $\alpha_{p}(t, L)<\left(1-\delta p^{(1-m) / 2}\right)^{-1}$.

Suppose $2 \mid e, e>0$; then $\alpha_{p}(t, L)$ is less than or equal to

$$
\begin{aligned}
(1- & \left.p^{1-m}\right)\left(1-p^{(2-m) e / 2}\right)\left(1-p^{2-m}\right)^{-1} \\
& +p^{(2-m) e / 2}\left(1-p^{1-m}\right)\left(1-p^{(1-m) / 2}\right)^{-1} \\
= & \left(1-p^{1-m}\right)\left(1-p^{2-m}\right)^{-1}\left(1-p^{(1-m) / 2}\right)^{-1} \\
& \times\left\{1-p^{(1-m) / 2}+p^{(1-m) / 2+(2-m) e / 2}-p^{(2-m)(e / 2+1)}\right\} \\
< & \left(1-p^{1-m}\right)\left(1-p^{2-m}\right)^{-1}\left(1-p^{(1-m) / 2}\right)^{-1}\left(1-p^{(2-m)(e / 2+1)}\right) \\
< & \left(1-p^{2-m}\right)^{-1}\left(1-p^{(1-m) / 2}\right)^{-1}
\end{aligned}
$$

Thus we have, for odd $m$

$$
\prod_{p \nmid 2 d L} \alpha_{p}(t, L)<\prod_{p \nmid 2 t d L}\left(1-\delta_{p} p^{(1-m) / 2}\right)^{-1} \cdot \prod_{p \mid t}\left(1-p^{2-m}\right)^{-1}\left(1-p^{(1-m) / 2}\right)^{-1} .
$$

Therefore for odd $m \geq 5$ we have $\prod_{p \nmid 2 d L} \alpha_{p}(t, L) \ll 1$, and for $m=3$, 


$$
\begin{aligned}
\prod_{p \nmid 2 d L} \alpha_{p}(t, L) & <\prod_{p \nmid 2 t d L}\left(1-\delta_{p} p^{-1}\right)^{-1} \cdot \prod_{p \mid t}\left(1-p^{-1}\right)^{\cdots 2} \\
& \ll(t d L)^{\varepsilon},
\end{aligned}
$$

which completes the proof of Lemma 8.

Lemma 9. For a natural number $t$ we have

$$
\alpha_{p}(t, L) \leq 2^{\delta_{2, p}}\left(1-p^{2-m}\right)^{-1} \max d_{p}(b, L),
$$

where $b$ runs over non-zero integers, $d_{p}$ denotes the primitive local density and $\delta$ is the Kronecker's delta function.

Proof. It is known [7], [2] that for $a \neq \equiv 0 \bmod p$ and $r \geq 0$,

$$
\begin{aligned}
\alpha_{p}\left(a p^{r}, L\right) & =2^{\delta_{2, p}} \sum_{0 \leq k \leq r / 2} p^{k(2-m)} d_{p}\left(a p^{r-2 k}, L\right) \\
& <2^{\delta_{2}, p}\left\{\max _{b} d_{p}(b, L)\right\} \sum_{k \geq 0} p^{k(2-m)} \\
& =2^{\delta_{2, p}}\left(1-p^{2-m}\right)^{-1} \max d_{p}(b, L) .
\end{aligned}
$$

Lemma 10. For a natural number $t$ we have

$$
\prod_{p} \alpha_{p}(t, L) \ll(t d L)^{\varepsilon} \prod_{p \mid 2 d L}\left\{\max _{0 \neq b \in Z} d_{p}(b, L)\right\}
$$

for any positive number $\varepsilon$.

Proof. By virtue of Lemmas 8, 9, we have

$$
\begin{aligned}
\prod_{p} \alpha_{p}(t, L) & \ll(t d L)^{\varepsilon} \prod_{p \mid 2 d L}\left(1-p^{2-m}\right)^{-1} \prod_{p \mid 2 d L}\left\{\max _{b} d_{p}(b, L)\right\} \\
& \ll t^{\varepsilon}(d L)^{2 \varepsilon} \prod_{p \mid 2 d L}\left\{\max d_{p}(b, L)\right\} .
\end{aligned}
$$

Lemma 11. For a natural number $t$ we have

$$
\prod_{p} \alpha_{p}(t, L) \ll(t d L)^{\varepsilon} \prod_{p \mid 2 d L} \sqrt{p^{a p}}
$$

where $\varepsilon$ is any positive number and $a_{p}$ is the integer defined in Theorem.

Proof. We have only to prove

$$
d_{p}(b, L)<C_{\varepsilon} p^{\varepsilon \operatorname{ord}_{p} d L+a_{p} / 2},
$$

where $C_{\varepsilon}$ depends only on $\varepsilon$, since $\prod_{p \mid 2 d L} C_{\varepsilon} \ll(d L)^{\varepsilon}$. Let $h$ be an integer such that $p^{n} n\left(L^{\#}\right) \subset 2 p Z_{p}$. It is known [2]

$$
d_{p}(b, L)=p^{\text {ord } d a L+h(1-m)} \# D\left(b, L ; p^{h}\right),
$$

where 


$$
D\left(b, L ; p^{h}\right)=\left\{x \in Z_{p} L / p^{h} Z_{p} L^{\#} \mid Q(x) \equiv b \bmod 2 p^{h} Z_{p}, x \notin p Z_{p}\right\} .
$$

Let an orthogonal splitting of $Z_{p} L$ be $L_{1} \perp \cdots \perp L_{s}$ where $L_{i}$ is $p^{a_{i}}$ modular for $i \geq 2$ and $a_{2} \leq \cdots \leq a_{s}$ and a Jordan splitting of $L_{1} \perp L_{2}$ gives a Jordan splitting of $Z_{p} L$; then we can put $h=a_{s}+2=O\left(p^{\varepsilon \operatorname{ord}_{p} d L}\right)$, and we have

$$
\begin{aligned}
& \# D\left(b, L ; p^{n}\right) \\
& \leq \sum_{\substack{x \in 1 L_{i} / p^{h-a_{i} L_{i}} \\
i \geq 2}} \sharp\left\{y \in L_{1} / p^{h} L_{1}^{\sharp} \mid Q(y) \equiv b-Q(x) \bmod 2 p^{h} Z_{p}\right\} \\
& \leq p^{\Sigma_{i \geq 2}\left(h-a_{i}\right) \text { rk } L_{i}} \max _{c \in Z} \sharp\left\{y \in L_{1} / p^{h} L_{1}^{\#} \mid Q(y) \equiv c \bmod 2 p^{h} Z_{p}\right\}
\end{aligned}
$$

and hence we have

$$
d_{p}(b, L) \leq p^{\operatorname{ord}_{p} d L_{1}+h\left(1-\mathrm{rk} L_{1}\right)} \max _{c \in Z} \#\left\{y \in L_{1} / p^{h} L_{1}^{\#} \mid Q(y) \equiv c \bmod 2 p^{h} Z_{p}\right\} .
$$

Suppose $Z_{p} L=\left\langle 2 \varepsilon_{1}\right\rangle \perp\left\langle 2 p^{a} \varepsilon_{2}\right\rangle \perp \cdots, \varepsilon_{1}, \varepsilon_{2} \in Z_{a}^{\times}, a \geq 0$ (Jordan splitting). We put $L_{1}=\left\langle 2 \varepsilon_{1}\right\rangle \perp\left\langle 2 p^{a} \varepsilon_{2}\right\rangle$; then we have

$$
\begin{aligned}
& \sharp\left\{y \in L_{1} / p^{h} L_{1}^{\#} \mid Q(y) \equiv c \bmod 2 p^{h} Z_{p}\right\} \\
= & \#\left\{u \bmod p^{h-\delta}, v \bmod p^{h-a-\delta} \mid 2 \varepsilon_{1} u^{2}+2 p^{a} \varepsilon_{2} v^{2} \equiv c \bmod 2 p^{h} Z_{p}\right\},
\end{aligned}
$$

where $\delta=\delta_{2, p}$

$=O\left(h p^{h-a / 2}\right)$ by Lemma 3. Thus we have

$$
d_{p}(b, L) \ll p^{a-h} \cdot h p^{h-a / 2}<h p^{a / 2} \ll p^{\varepsilon \operatorname{ord}_{p} d L+a / 2} .
$$

Next we suppose that $p=2$ and $Z_{2} L=\langle 2 \varepsilon\rangle \perp\left\langle 2^{a}\left(\begin{array}{cc}2 d & 1 \\ 1 & 2 d\end{array}\right)\right\rangle \perp \cdots$, $\varepsilon \in Z_{p}^{\times}, a \geq 2, d=0,1$. Putting $L_{1}=\langle 2 \varepsilon\rangle \perp\left\langle 2^{a}\left(\begin{array}{cc}2 d & 1 \\ 1 & 2 d\end{array}\right)\right\rangle$, we have

$$
\sharp\left\{y \in L_{1} / p^{h} L_{1}^{\#} \mid Q(y) \equiv c \bmod 2^{h+1} Z_{2}\right\}
$$

$=\#\left\{u \bmod 2^{h-1}, v, w \bmod 2^{h-a} \mid 2 \varepsilon u^{2}+2^{a+1}\left(d v^{2}+v w+d w^{2}\right) \equiv c \bmod 2^{h+1}\right\}$

$\ll h \cdot 2^{2 h-3 a / 2} \quad$ (by Lemmas 6,7 ).

Hence we have $d_{2}(b, L) \ll 2^{1+2 a-2 h} \cdot h \cdot 2^{2 h-3 a / 2} \ll 2^{a / 2+\varepsilon \operatorname{ord}_{2} d L}$ as above.

Lastly we suppose $p=2$ and $Z_{2} L=\left\langle\left(\begin{array}{cc}2 d & 1 \\ 1 & 2 d\end{array}\right)\right\rangle \perp \cdots, d=0$ or 1 by which we exhaust all types of Jordan splittings. Putting $L_{1}=\left\langle\left(\begin{array}{cc}2 d & 1 \\ 1 & 2 d\end{array}\right)\right\rangle$, we have

$$
\begin{aligned}
& \sharp\left\{y \in L_{1} / 2^{h} L_{1}^{\sharp} \mid Q(y) \equiv c \bmod 2^{h+1} Z_{2}\right\} \\
= & \#\left\{u, v \bmod 2^{h} \mid 2\left(d u^{2}+u v+d v^{2}\right) \equiv c \bmod 2^{h+1}\right\} \\
\ll & \left.h \cdot 2^{h} \quad \text { (by Lemmas } 4,5\right) .
\end{aligned}
$$


Therefore we have $d_{2}(b, L) \ll 2^{-h} \cdot h \cdot 2^{h} \ll 2^{\varepsilon \operatorname{ord}_{2} d L}$, and it completes the proof of Lemma.

Now we can prove Theorem, following an idea due to Conway, Thompson on p. 46 in [7]. Put

$$
w(M)=\left\{\sum_{N \in \operatorname{gen} L}(\# O(N))^{-1}\right\}^{-1} \cdot(\# O(M))^{-1}
$$

and

$$
r(t, \text { gen } L)=\sum_{N \in \operatorname{gen} L} w(N) r(t, N)
$$

where $N$ 's run over representatives of isometry classes in the genus of $L$ and $O(N)$ is the group of isometries of $N$ and $r(t, N)=\#\{x \in N \mid$ $Q(x)=t\}$. It is known [9] that $r(t$, gen $L)=c(d L)^{-1 / 2} t^{m / 2-1} \prod_{p} \alpha_{p}(t, L)$ for some constant $c$ and hence we have

$$
\begin{aligned}
\sum_{t=1}^{k} r(t, \text { gen } L) & \ll(d L)^{-1 / 2} \sum_{t=1}^{k} t^{m / 2-1}(t d L)^{\varepsilon} \prod_{p \mid 2 d L} \sqrt{p^{a_{p}}} \quad \text { (by Lemma 11) } \\
& \ll(d L)^{\varepsilon-1 / 2} \prod_{p \mid 2 d L} \sqrt{p^{a_{p}}} \cdot k^{m / 2+\varepsilon} .
\end{aligned}
$$

Suppose $\sum_{x=1}^{k} r(t, M)>0$ for every $M$ in gen $L$; then we have

$$
\sum_{t=1}^{k} r(t, \operatorname{gen} L)=\sum_{M \in \operatorname{gen} L} w(M) \sum_{t=1}^{k} r(t, M) \geq \sum_{M \in \operatorname{gen} L} w(M)=1,
$$

and hence $k^{m / 2+\varepsilon} \gg(d L)^{1 / 2-\varepsilon} \prod_{p \mid 2 d L} \sqrt{p^{-a_{p}}}$. Therefore $k=C_{\varepsilon}(d L)^{(1 / 2-\varepsilon) /(m / 2+\varepsilon)}$ - $\left(\prod_{p \mid 2 d L} p^{-a_{p}}\right)^{1 /(m+2 \varepsilon)}$ for some $C_{\varepsilon}$ is contradictory for any positive number $\varepsilon$. Thus $\sum_{t=1}^{k} r(t, M)=0$ holds for some $M \in$ gen $L$ and the above $k$ and this yields $\min M>k$. Since $(1 / 2-\varepsilon) /(m / 2+\varepsilon)$ tends to $1 / m$ from below as $\varepsilon \rightarrow 0$ and $-(m+2 \varepsilon)^{-1}>-m^{-1}$, this means

$$
\min M \gg(d L)^{1 / m-\varepsilon}\left(\prod_{p \mid 2 d L} p^{a_{p}}\right)^{-1 / m} \quad \text { for any } \varepsilon>0
$$

and completes the proof of Theorem.

\section{REFERENCES}

[1] J. S. Hsia, Y. Kitaoka, M. Kneser, Representations of positive definite quadratic forms, J. reine angew. Math., 301 (1978), 132-141.

[2] Y. Kitaoka, Modular forms of degree $n$ and representation by quadratic forms II, Nagoya Math. J., 87 (1982), 127-146.

[3] - Lectures on Siegel modular forms and representation by quadratic forms, Tata Institute of Fundamental Research, Bombay, Berlin-Heidelberg-New York, Springer 1986. 
[ 4 ] - Local densities of quadratic forms, In : Investigations in Number Theory, 1987 (Advanced Studies in Pure Math. 13, pp. 433-460).

[5] - - A note on representation for positive definite binary quadratic forms by positive definite quadratic forms in 6 variables, to appear,

[ 6 ] - Modular forms of degree $n$ and representation by quadratic forms $\mathrm{V}$, Nagoya Math. J., 111 (1988), 173-179.

[ 7 ] J. Milnor, D. Husemoller, Symmetric bilinear forms, Berlin-Heidelberg-New York, Springer 1973.

[ 8 ] O. T. O'Meara, Introduction to quadratic forms, Berlin-Heidelberg-New York, Springer 1963.

[9 ] C. L. Siegel, Über die analytische Theorie der quadratischen Formen, Ann. of Math., 36 (1935), 527-606.

Department of Mathematics

School of Science

Nagoya University

Chikusa-ku, Nagoya 464

Japan 